\title{
4 ADHS und bipolare Störungen im Kindes- und Jugendalter
}

Hellmuth Braun-Scharm

\subsection{Einleitung und Geschichte}

Die Diskussionen der letzten Jahre über einen möglichen Zusammenhang zwischen ADHS und bipolaren Störungen sowie das Überwiegen der englischsprachigen Literatur haben mancherorts zu der irrigen Meinung geführt, dass der Begriff bipolar aus dem amerikanischen stammt (s. Tab. 1). Aus historischer Sicht stammt das Konzept der zyklischen (oder auch phasischen, episodischen, bipolaren) Störungen aber aus der französischen Psychiatrie des 19. Jahrhunderts und wurde von Falret und Baillarger beschrieben (Angst und Marneros 2001).

Tab. 1 Historische Meilensteine des Konzeptes der bipolaren Störungen

\begin{tabular}{l|l}
\hline IP Falret $(1851,1854)$ & Folie circulaire \\
\hline $\begin{array}{l}\text { J. Baillarger }(1854) \\
\text { K. Kahlbaum }(1863,1882)\end{array}$ & Folie ä double forme \\
\hline E. Mendel $(1881)$ & cyclisches Irresein \\
\hline E. Kraepelin $(1893,1896)$ & Hypomanie \\
\hline K. Kleist $(1911)$ & Konzept Manie-Depression \\
\hline J. Angst, C Perris (1966) & Wiedergeburt der BIP \\
\hline DL Dunner et al. (1976) & Bipolar II \\
\hline HS Akiskal, G. Mallya (1987) & soff bipolar spectrum \\
\hline HS Akiskal (1992) & mixed states \\
\hline
\end{tabular}


Kahlbaum transponierte dieses Konzept dann nach Deutschland und in der Tradition von Kraepelin wurde es zum Konzept der manisch- depressiven Psychosen (als dichotomer Gegenpol zu den schizophrenen Psychosen). Der Begriff bipolar und das Konzept der Polarität mit unipolaren und bipolaren Störungen stammt aus der Schule von Wernicke, Kleist und Leonhardt. Angst und Perris haben mit zwei unabhängig voneinander im Jahr 1966 erschienenen Monographien zur „Wiedergeburt“ der bipolaren Störungen geführt und seitdem ist in der Psychiatrie des Erwachsenenalters durch erhebliche Forschungsaktivität eine große Zahl wichtiger Befunde und Innovationen zustande gekommen. Dazu gehört insbesondere die Etablierung der bipolar-II-Störung (Kombination von major depression und Hypomanie) als Ergänzung zur klassischen manisch-depressiven bipolar-I-Störung (Kombination von major depression und Manie). Der Züricher Langzeitstudie von Angst et al. ist es u. a. zu verdanken, wenn wir heute als gesichert annehmen, dass sich zusätzlich zu den eigenständigen bipolar-II-Patienten auch unter den primär als depressiv diagnostizierten Patienten ein zuerst unerkannter Anteil von bipolar-II-Patienten befindet. Die Hinzunahme der bipolar-II-Störung zur bipolar-I-Störung führt zu einer Erweiterung des bipolaren Spektrums sowie zu einer Erhöhung von Inzidenz und Prävalenz von bipolaren Störungen in epidemiologischen Untersuchungen (zu Ungunsten der Depressionen) (Angst et al. 2002).

Auch wenn die Publikationen über bipolare Störungen in den letzten Jahren zunehmend von angloamerikanischen Arbeiten dominiert werden, kann also festgehalten werden, dass die Ursprünge und die Tradition, aber auch zahlreiche neue Befunde der bipolaren Störungen fest in der europäischen Psychiatrie verwurzelt sind.

\subsection{Bipolare Störungen des Kindesalters}

In Bezug auf die ADHS haben die bipolaren Störungen allerdings in ganz anderer Form und Richtung Bedeutung gewonnen, und zwar vorwiegend in Bezug auf das Kindesalter (Biedermann et al. 1996, National Institute 2001). Insbesondere die Arbeitsgruppen von Geller und Biederman (Übersicht siehe Braun-Scharm 2005) haben in einer Vielzahl von Publikationen beschrieben, dass ein Teil der ADHS-Kinder durch eine spezifische Ausformung der Symptomatik abgegrenzt werden kann und dann möglicherweise einen eigenen bipolaren Subtyp, den so genannten Pediatric bipolar phenotype darstellt. Dabei stammen die Symptome, die zur Differenzierung dieser Subgruppe dienen, nahezu ausschließlich dem manischen Teil der bipolaren Störungen:

- Hypersexualität,

- Grandiosität,

v vermindertes Schlafbedürfnis.

Zusammen mit hoher Frequenz und kurzer Dauer der Schwankungen (ultradianes cycling nach Geller) ergibt sich ein kontinuierliches chronisches Krankheitsbild von hohem Schweregrad, das sich phenomenologisch nicht mehr 
mit den klassischen bipolaren Störungen vergleichen lässt, deren Hauptcharakteristik in der Kombination von abgegrenzten manischen (hypomanen) und depressiven Phasen besteht.

Dieser Vorstoß war aus europäischer Sicht umso erstaunlicher, als bipolare Störungen des Kindesalters bislang weder in der Diagnostik noch in der Therapie eine nennenswerte Rolle gespielt hatten. Die lange Reihe unterschiedlicher, kontroverser und unversöhnlich erscheinender diagnostischer Konventionen in den USA und Europa schien in Form der ADHS-Bipolar-Debatte ein neues Exempel gefunden zu haben. Die zugrunde liegenden nosologischen Fragestellungen lauten:

1. Gehören ADHS und bipolare Störungen zur selben nosologischen Entität, die sich nur durch Manifestationen in unterschiedlichen Altersgruppen unterscheidet?

2. Sind ADHS und bipolare Störungen komorbide Störungen, die häufig gemeinsam auftreten und nur an den extremen Alterspunkten (Kindheit, höheres Erwachsenenalter) solitär sind?

3. Sind ADHS und bipolare Störungen Extreme eines dimensionalen Schweregradspektrums, das im leichteren Falle ADHS, und im schweren FaIle, „Bipolar" heißt?

4. Cibt es eine gemeinsame genetische Wurzel von ADHS und bipolaren Störungen, sodass Kinder von bipolaren Eltern häufiger ADHS haben als andere Kinder?

Alle diese Fragen waren bisher nicht schlüssig zu beantworten und es schien, als ob die Debatte um ADHS und bipolare Störungen zu einer endlosen Kontroverse führen könnte. Anfang des Jahres 2007 erschien jedoch eine wegweisende Publikation, in der von amerikanischer Seite in selbstkritischer Weise zu diesem Thema Stellung genommen wurde (Practice Parameter 2007). Die in dieser Publikation vorgenommenen Formulierungen könnten dazu führen, dass in Zukunft US-amerikanische und europäische Kinder- und Jugendpsychiater auf der gleichen oder zumindest einer sehr ähnlichen Crundlage miteinander diskutieren können. Die wesentlichen Positionen dieser Publikation sind:

\subsection{Practice Parameter}

1. Als gesichert kann angenommen werden, dass es im Jugendalter Frühmanifestationen bipolarer Störungen gibt, die weitgehend den klassifikatorischen Kriterien im Erwachsenenalter entsprechen.

2. Über eine eigenständige bipolare Störung des Kindesalters (Pedriatric bipolar phednotyp) besteht kein Konsens, und zwar weder in Bezug auf diagnostische, prognostische noch therapeutische Gesichtspunkte. Insbesondere die Art der Kombination von ADHS und bipolarer Störung ist weiterhin ungeklärt.

3. Weiter weisen die bislang vorliegenden epidemiologischen Studien zu bipolaren Störungen im Kindesalter daraufhin, dass die Prävalenz solcher 
Störungsbilder mit etwa $1 \%$ recht niedrig ist und weit unter der Häufigkeit der ADHS-Diagnosen liegt (Holtmann et al. 2007, Hudziak et al. 2005, Lewinsohn et al. 2000).

Der Verdienst der amerikanischen Forschergruppen besteht ohne Zweifel darin, darauf hingewiesen zu haben, dass es in dem heterogenen Spektrum der ADHS schwere Ausprägungen gibt, die bisher unzureichend klassifiziert sind und transkontinental unterschiedlich zugeordnet werden. Eine Nähe zu den maniformen Ausprägungen des bipolaren Spektrums ist möglich, jedoch nicht zweifelsfrei erwiesen. Ob es sinnvoll ist, diese besonderen Verlaufformen von ADHS als bipolare Störungen zu klassifizieren, ist weiterhin fraglich.

\section{Bipolare Störungen des Jugendalters}

Auch wenn bipolare Störungen des Jugendalters unabhängig davon, ob sie dem bipolar-I oder bipolar-II-Typ angehören, weitgehend den Kriterien des Erwachsenenalters entsprechen, weisen sie dennoch einige alterstypische Besonderheiten auf:

\section{Jugendtypische Besonderheiten}

1. Differentialdiagnostische Abgrenzung zu den alterstypischen „physiologischen“ „pubertären“ Schwankungen

2. Häufiges Auftreten von rapid cycling mit folgenden Untergruppen:

- Rapid cycling 4 Episoden/Jahr

- Ultrarapid cycling vier Episoden/Monat

- Ultradian cycling mehrfache tägliche Episoden

3. Mixed states: manisch - hypomane und depressive Symptome gemischt oder in raschem Wechsel

4. Untypische Bilder mit Dysphorie, Irritierbarkeit und störendem Sozialverhalten

\subsection{Rapid cycling und Prognose}

Im Erwachsenenalter weisen manische oder depressive Episoden (unabhängig vom bipolaren Subtyp) meist eine Dauer von Wochen oder Monaten auf. In der Regel (nicht immer) werden die Phasen durch Intervalle von weitgehender Remission unterbrochen, sodass sich die Patienten erholen und ihren bisherigen Lebensweg fortsetzten können. Auch im Erwachsenenalter gibt es die Verlaufsform des rapid cycling, die durch kürzere Phasen und schnellere Wechsel gekennzeichnet ist. Seit Langem wird die Hypothese vertreten, dass rapid cycling im Jugendalter häufiger ist. Eine alternative Hypothese könnte lauten, dass unabhängig von der Phasendauer das Ausmaß der Remission im Jugendalter geringer ist als im Erwachsenenalter, sodass es häufiger zu quasi-chronischen Krankheitsverläufen kommt. 
Abgesehen von vereinzelten Publikationen unterschiedlicher Güte spricht auch die eigene klinische Erfahrung dafür, dass gemischte Zustände (manisch-depressive und andere gemischte Zustände) häufige Symptome bipolarer Störungen im Jugendalter sind (Birmaher et al. 2006). Gemischte manische-depressive, im weitesten Sinne zyklothyme, zeitweise auch agitierte Zustände sind sowohl für die Patienten als auch für ihr Umfeld Phasen erhebliche Unruhe, die durchaus eine gewisse Ähnlichkeit mit hyperkinetischen Zuständen des Kindesalters aufweisen, jedoch normalerweise nicht auf $\mathrm{Me}$ thylphenidat, Amphetaminpräparate oder Atomoxetin ansprechen und bei längerer Beobachtung doch eine gewisse Phasenhaftigkeit erkennen lassen. Für diese Annahme spricht weiterhin eine Bemerkung von Leonhardt, der gemischte Zustände auch als typisch für die bipolaren Störungen des Erwachsenenalters beschrieb (Leonhard 1986).

Die Kombination von kürzerer Phasendauer, höherer Phasenfrequenz und gemischten Zuständen kann auch im Jugendalter bereits zu einem eher chronischen klinischen Bild führen, das zu erheblichen psychosozialen Einschränkungen führt, auch wenn die charakteristischen psychotischen Symptome fehlen. Eine geregelte Schullaufbahn oder Ausbildung ist unmöglich, wenn immer wieder behandlungsbedürftige psychopathologische Zustände interferieren, deren Behandlung schwierig und von wenig vorhersehbarer Dauer ist. Im Gegensatz zu den klassischen Verlaufsformen im Erwachsenenalter haben deshalb bipolare Jugendliche mit den zuletzt beschriebenen Krankheitsbildern eine durchaus ernste Prognose und bedürfen einer langfristigen, intensiven sozialpsychiatrischen und medikamentösen Begleitung, um der frühzeitigen Invalidisierung zu entgehen.

\subsection{Therapie}

Die Behandlung von ADHS und bipolarer Störung unterscheidet sich grundsätzlich.

Wenn bei der ADHS der Einsatz von Medikation (Methylphenidat, Amphetamine, Atomotexin etc.) und psychotherapeutischen Interventionen (Elterntraining, Konzentrationstraining, Hausaufgabenbetreuung etc.) im optimalen Falle eine rasche und anhaltende Besserung erbringt, unterscheidet sich die Behandlung von bipolaren Störungen in mehrfacher Hinsicht und ist nicht weniger vielfältig und schillernd als das Krankheitsbild selbst. In medikamentöser Hinsicht kommen vor allem bei der Langezeitmedikation (Phasenprophylaxe) andere vorwiegend stimmungsstabilisierende Substanzgruppen zum Einsatz (Lithiumsalze, Antikonvulsiva). In der Akutbehandlung dominieren hingegen Neuroleptika neuerer Art (z. B. Quetiapin, Risperidon, Ziprasidon, Aripiprazol). Krankheitseinsicht, Kooperation, regelmäßige Medikamenteneinnahme und Teilnahme an psychotherapeutischen Maßnahmen sind bei bipolaren Störungen noch weniger ausgeprägt als bei ADHS. Grundsätzlich gilt, dass eine multimodale Behandlung unter Einschluss medikamentöser 
Ansätze bei beiden Krankheitsbildern sinnvoll ist oder sinnvoll wäre, aber häufig aufgrund mangelnder Akzeptanz und Compliance nicht im gewünschten Umfang durchführbar ist. Die US-amerikanische Haltung, therapieresistente ADHS-Kindern mit Medikamenten aus dem bipolaren Spektrum zu behandeln (Lithium, Antikonvulsiva, Neuroleptika) muss solange kritisch betrachtet werden, solange deren Wirksamkeit nicht erwiesen ist. Andererseits wirkt die zurückhaltende therapeutische Haltung europäischer Kinder- und Jugendpsychiater angesichts schwieriger ADHS-Kinder bisweilen wenig kreativ. Ungeachtet der theoretischen Debatte sollte das vorhandene Spektrum von Medikamenten unter strenger Beachtung einer überprüfbaren Behandlungshypothese in Betracht gezogen werden. In einzelnen Fällen haben wir z. B. gute Erfahrungen mit der alleinigen Gabe von Quetiapin oder Ziprasidon gemacht, die vorher jahrelang erfolglos mit Methylphenidat und AmphetaminPräparaten behandelt worden waren. Eine gewisse Einschränkung besteht darin, dass die Mehrzahl der genannten Substanzen noch nicht für Kinder zugelassen ist und daher „off labet“ eingesetzt werden muss (nach ausdrücklichem Einverständnis der Sorgeberechtigten).

\section{Schlussbemerkung}

Die transkontinentale Kontroverse über einen möglichen Zusammenhang von ADHS und bipolarer Störung ist nach wie vor ungelöst. Die Entwicklung von Forschung und klinischer Erfahrung wird sicher weitere Klarheit bringen. 


\section{Literatur}

Angst I, Gamma A, Lewinsohn P, The evolving epidemiology of bipolar disorder. Word Psychiatry 2002; 1 (3): 146-8.

Angst I, Marneros A, Bipolarity from ancient to modern timest conception, birth and rebirth. Journal Affect Disorders 2001; (67), 3-19.

Biedermann J, Faraone S, et al., Attention-deficit hyperacitivity disorder and juvenile mania: an overlooked comorbidity? Journal of Am. Acad. Child Adolesc. Psychiatry 1996; 35 (8), 997-1008.

Birmaher B, Axelson D, Strober M, Gill MK, Valeri S, Chiappetta L, Ryan N, Leonard H, Hunt I, lyengar S, Keller $\mathrm{M}$, Clinical course of children and adolescents with bipolar spectrum disorders. Arch Gen Psychiatry 2006; (63): 175-183.

Braun-Scharm H, Leeners |, Schmidhauser |, Bipolare affektive Störungen im Kindesund Jugendalter- eine Übersicht. Forum der Kinder- und Jugendpsychiatrie und Psychotherapie. Forum-Verlag 2005; Heft 2, S 11-50.

Geller B, Delbello M, Bipolar Disorder in Childhood and Early Adolescence. The Guilford Press 2003.

Holtmann M, Goth K, Wöckel L, Poustka F, Bölte S, CBCL-pediatric bipolar disorder phenotype: severe ADHD or bipolar disorder? Journal of Neural Transmission (in press) 2007; 1-7.

Hudziak II, Althoff RR, Derks EM, Faraone SV, Boomsma DI, Prevalence and genetic architecture of child behavior checklist-juvenile bipolar disorder. Biol. Psychiatry 2005; (58): 562-568.

Leonhard K, Aufteilung der endogenen Psychosen und ihre differenzierte Ätiologie, Akademie-Verlag Berlin 1986; 6. Auflage.

Lewinsohn PM, Klein DN, Seeley JR, Bipolar disorder during adolescence and young adulthood in a community sample. Bipolar Disord 2000; (2): 281-293.

National Institute of Mental health research roundtable an prepubertal bipolar disorder. Journal Am. Acad. Child Adolesc. Psychiatry 40, 2001; (8): 871-878.

Practice Parameter for the assessment and treatment of children and adolescents with bipolar disorder. Journal Am. Acad. Child Adolesc. Psychiatry 46, 2007; (1): 107-125. 